\title{
Investigation on psychosomatic status of entry quarantine personnel during the COVID-19 pandemic
}

\author{
Shi-Hua SHEN ${ }^{1}$, Yun-Kai HU², Xian-Gui RAN ${ }^{1}$, Zhen-Hua ZHU'1, Hong-Bo LIU ${ }^{1}$, Jia-Liang WANG ${ }^{1}$, Qian HONG ${ }^{3}$, \\ Rong-Tao $\mathrm{WU}^{4 *}$ (D)
}

\begin{abstract}
To investigate the anxiety, depression, fatigue and insomnia of the entry quarantine personnel during the COVID-19 pandemic, and the influencing factors of psychological status were also explored. The Self-Rating Anxiety Scale (SAS), Self-Rating Depression Scale (SDS), Fatigue severity scale (FSS) and the Insomnia Severity Index (ISI) of 630 entry quarantine personnel were performed and the correlation analysis of the four types of scales was performed. The incidence rates of anxiety, depression, fatigue and insomnia were $4.76 \%, 20.00 \%, 48.57 \%$ and $22.86 \%$, respectively. There was a positive correlation between the four indicators. Univariate analysis showed that age, education level, occupation, continent and reasons for going abroad were the main influencing factors of psychosomatic status. The lower the age group, the higher the education level, and the healthier the psychosomatic health of the entry quarantine personnel. The psychological status of international students was healthier than that of the staff. The psychological condition of the quarantined people in Asia was healthier than that in other continents. 630 entry quarantine personnel had different degrees of anxiety, depression, fatigue and insomnia, there was a positive correlation between them. Age, education level, occupation, continent and reasons for going abroad were the main influencing factors of psychological status.
\end{abstract}

Keywords: COVID-19; entry quarantine personnel; psychological status; questionnaire.

Practical Application: This study takes the entry quarantine personality as the observation population, focusing on the changes of psychological state of such people in the face of major public health emergencies, in order to effectively reduce the psychological damage of isolation and improve compliance; Through the analysis of the characteristics and laws of mental health and mental unhealthy, it is convenient to adopt targeted measures to protect the mental health of isolated personnel, reduce the adverse effects of isolation measures, and even reduce the psychological panic of the whole population.

\section{Introduction}

Novel Coronavirus Disease 2019 (COVID-19), as an acute infectious respiratory disease with global pandemic characteristics, has become a major global public health event (Yang et al., 2020). The World Health Organization (WHO) has declared that the outbreak of COVID-19 in China is a public health emergency of international concern (PHEIC) (Eurosurveillance Editorial Team, 2020; Li et al., 2020a). With the valiant efforts of the whole country, China has achieved initial results in the epidemic situation, but the epidemic outside China is intensifying and spreading. The number of infected people in some countries and regions has risen sharply, and various clustering events caused by imported cases have attracted great concern in China (China, 2020b; Yuan et al., 2020). Since March 5, 2020, when imported cases were included in the epidemic statistics, "External Anti Input" has gradually become the main body of epidemic prevention and control at the present stage in China.
Due to the outbreak of COVID-19, people inevitably have suffered from many psychological problems. Domestic and foreign scholars have studied the psychological status of medical workers, domestic quarantine personnel and other groups, but there is a lack of investigation and understanding of the psychological status of entry quarantine personnel (non-Chinese nationality). Therefore, in order to explore the psychological status of entry quarantine personnel and the influencing factors, we carried out a special investigation among entry quarantine personnel who entering Fuyang City.

\section{Materials and methods}

\subsection{Subjects}

A total pf 630 entry quarantine personnel who entered Fuyang City from March 5 to March 31, 2020 were enrolled in this study. Inclusion criteria: (1) People who came from 
overseas, including Hong Kong, Macao and Taiwan; (2) People who were informed consent and voluntary participation in the study. Exclusion criteria: People entering Fuyang from other provinces in China.

\subsection{Research tools and methods}

The following contents were included and self-made questionnaire through questionnaire star, and link is generated and shared with those entry quarantine personnel. The personnel answer and submit questionnaire through their own mobile phone.

(1) General information, including country, gender, age, occupation, education level, reason for going abroad, quarantine point, overseas living time, whether they have been in contact with patients with COVID-19; (2) Anxiety and depression status, which were evaluated by using Self-Rating Anxiety Scale (SAS) and Self-Rating Depression Scale (SDS); (3) Fatigue and sleep status, which were evaluated by using fatigue severity scale (FSS) and the Insomnia Severity Index (ISI).

\subsection{Scoring criteria}

SAS consists of 20 items, which are divided into 4 grades. The score below 50 points is normal; 50-60 is mild anxiety; $61-70$ is moderate anxiety; and more than 70 is severe anxiety. SDS contains 20 items, which are divided into 4 grades. The score below 50 points is normal; 51-60 is mild depression, 61-71 is moderate depression, and more than 70 is severe depression (Wang et al., 1999). FSS score below 36 points indicates that people may not feel fatigue, and a score of greater than or equal to 36 indicates that people may need a doctor for further evaluation (Wei \& Song, 2017). ISI score of 0 to 7 points indicates no clinically significant insomnia, 8 to 14 indicates sub-threshold insomnia, 15 to 21 indicates moderate and severe clinical insomnia, 22 to 28 indicates severe clinical insomnia (Li, 2018).

\subsection{Statistical analysis}

Statistical software SPSS 21.0 was used for statistical analysis of the collected data. The measurement data were described as means \pm standard deviation (SD). The count data were described as percentages. The comparison between groups was conducted by t-test and one-way ANOVA. Least significant difference (LSD) method was used for pairwise comparison. Pearson correlation analysis was used for anxiety, depression, fatigue and insomnia scores. $P \leq 0.05$ was the difference with statistical significance.

\section{Results}

\subsection{Baseline data}

From March 5 to March 31, 2020, a questionnaire survey was conducted on 652 entry quarantine personnel in Fuyang City. A total of 630 questionnaires were returned, with a recovery rate of $96.63 \%$. Among the 630 entry quarantine personnel, 384 were males $(60.95 \%)$ and 246 were females $(39.05 \%)$. There were 318 subjects ( $50.48 \%$ ) between $20-29$ years old, and $180(28.57 \%)$ had a bachelor degree. In terms of occupation, the number of employees $(342,54.29 \%)$ was slightly more than international students $(288,45.71 \%)$. Hotels were the most concentrated quarantine points, with a majority of $402(63.81 \%)$. Most of the entry quarantine personnel were as international students and returned to China, accounting for 288 (45.71\%). Most of the entry quarantine personnel live abroad within one year.

As shown in Table 1, among the 630 subjects, the majority were Asian (306, 48.57\%), followed by 198 cases $(31.43 \%)$ in Europe, and 24 cases $(3.81 \%)$ in Oceania have the smallest proportion. The composition of entry quarantine personnel in different countries on the same continent was different. Among them, 150 subjects $(23.81 \%)$ in the UK in Europe was higher than that of other countries in the European region. Meanwhile, the UK was the country with the highest proportion of entry quarantine personnel. Moreover, Cambodia in Asia, the USA in the Americas and Nigeria in Africa had a higher composition of entry quarantine personnel than other countries or regions in their continent. In Oceania, only 24 cases (3.81\%) came from Australia.

\subsection{Incidence of anxiety, depression, fatigue and insomnia among entry quarantine personnel}

As shown in Table 2, among the 630 entry quarantine personnel, the incidence of fatigue was the highest (48.57\%),

Table 1. Composition of quarantine personnel from different continents.

\begin{tabular}{|c|c|c|c|c|c|c|c|}
\hline Continent & Country & Number of cases & $\begin{array}{c}\text { Constituent ratio } \\
(\%)\end{array}$ & Continent & Country & Number of cases & $\begin{array}{c}\text { Constituent ratio } \\
(\%)\end{array}$ \\
\hline \multirow{12}{*}{ Asia (306 cases) } & Cambodia & 84 & 13.33 & \multirow{4}{*}{$\begin{array}{c}\text { Europe } \\
\text { (198 cases) }\end{array}$} & Britain & 150 & 23.81 \\
\hline & Thailand & 48 & 7.62 & & France & 27 & 4.29 \\
\hline & Macao, China & 36 & 5.71 & & Germany & 15 & 2.38 \\
\hline & Myanmar & 30 & 4.76 & & Switzerland & 6 & 0.95 \\
\hline & Philippines & 24 & 3.81 & \multirow{3}{*}{$\begin{array}{l}\text { America } \\
\text { (72 cases) }\end{array}$} & USA & 60 & 9.52 \\
\hline & Laos & 24 & 3.81 & & Canada & 7 & 1.11 \\
\hline & Malaysia & 21 & 3.33 & & Uruguay & 5 & 0.79 \\
\hline & Singapore & 17 & 2.70 & \multirow{3}{*}{ Africa (30 cases) } & Nigeria & 12 & 1.90 \\
\hline & Pakistan & 8 & 1.27 & & Tanzania & 12 & 1.90 \\
\hline & Bangladesh & 5 & 0.79 & & Ethiopia & 6 & 0.95 \\
\hline & Japan & 5 & 0.79 & \multirow{2}{*}{$\begin{array}{c}\text { Oceania } \\
\text { (24 cases })\end{array}$} & \multirow{2}{*}{ Australia } & \multirow{2}{*}{24} & \multirow{2}{*}{3.81} \\
\hline & Viet Nam & 4 & 0.63 & & & & \\
\hline
\end{tabular}


Table 2. Anxiety, Depression, Fatigue and Insomnia in entry quarantine personnel.

\begin{tabular}{|c|c|c|c|}
\hline \multicolumn{2}{|c|}{ Items } & \multirow{2}{*}{$\frac{\text { Number }}{600}$} & \multirow{2}{*}{$\begin{array}{c}\text { Incidence (\%) } \\
4.76\end{array}$} \\
\hline Anxiety & Normal & & \\
\hline & Mild anxiety & 30 & \\
\hline \multirow[t]{4}{*}{ Depression } & Normal & 504 & 20.00 \\
\hline & Mild depression & 72 & \\
\hline & Moderate depression & 48 & \\
\hline & Severe depression & 6 & \\
\hline \multirow[t]{2}{*}{ Fatigue } & Normal & 324 & 48.57 \\
\hline & Fatigue & 306 & \\
\hline \multirow[t]{4}{*}{ Insomnia } & No clinically significant insomnia & 486 & 22.86 \\
\hline & Subliminal insomnia & 114 & \\
\hline & $\begin{array}{c}\text { Moderate to severe clinical } \\
\text { insomnia (moderate to severe) }\end{array}$ & 24 & \\
\hline & Severe clinical insomnia & 6 & \\
\hline
\end{tabular}

Table 3. Correlation analysis of anxiety, depression, fatigue and insomnia among entry quarantine personnel ( $P$ value).

\begin{tabular}{lccc}
\hline & Depression score & Fatigue score & Insomnia score \\
\hline Anxiety score & $0.773^{*}$ & $0.278^{*}$ & $0.575^{*}$ \\
Depression score & & $0.291^{*}$ & $0.592^{*}$ \\
Fatigue score & & $0.398^{*}$ \\
\hline "Represents significant correlation at 0.01 level (bilateral).
\end{tabular}

the incidence of sleep and depression were $22.86 \%$ and $20.00 \%$, respectively. The incidence of anxiety was the lowest (4.76\%).

\subsection{The incidence of anxiety, depression, fatigue and insomnia among entry quarantine personnel in different continents}

The incidences of anxiety, depression, fatigue and insomnia were different in different continents. Among them, the incidences of anxiety in entry quarantine personnel from the Europe and Asia were $6.06 \%$ and $3.92 \%$, respectively. However, those subjects from the Americas, Africa and Australia had not anxious. The incidences of depression in entry quarantine personnel from the Africa, Asia, Australia and Europe were $40.00 \%, 29.41 \%, 25.00 \%$, and $9.09 \%$, respectively. Those from the Americas did not experience depression. The entry quarantine personnel from Asia, Europe, Americas, Africa, and Oceania all experienced fatigue, and the rates were $20.95 \%, 14.29 \%$, $7.62 \%, 2.86 \%, 2.86 \%$, respectively. The incidence of insomnia among entry quarantine personnel from Asia, Europe, Africa, and Oceania was $15.24 \%, 5.71 \%, 0.95 \%$ and $0.95 \%$, respectively. Those from the Americas did not experience insomnia.

The incidence rates of anxiety and depression among the entry quarantine personnel from different countries were also not consistent. The incidence of anxiety and depression of entry quarantine personnel from the Macao of China was higher than that in other countries, the incidence of depression in Pakistan was higher than that from other countries, and the incidences of fatigue and insomnia in the British were higher than those of other countries. Some entry quarantine personnel from the Britain, Macao, China and Myanmar had different degrees of anxiety, depression, fatigue and insomnia, as shown in Figure 1 and Figure 2.

\subsection{Correlation analysis of anxiety, depression, fatigue and insomnia among entry quarantine personnel}

The correlation of anxiety score, depression score, fatigue score and insomnia score of 630 entry quarantine personnel were analyzed by Pearson correlation analysis. There were positive correlations between anxiety score and depression score, fatigue score and insomnia score $(0.773,0.278,0.575)$. There were also positive correlations between depression score and fatigue score, insomnia score $(0.291,0.592)$. There was also positive correlation between fatigue and insomnia scores (0.398) (Table 3).

\subsection{Univariate analysis of anxiety, depression, fatigue and insomnia among entry quarantine personnel}

The survey results showed that there were significant differences in anxiety scores among subjects with different occupations, continents and reasons for going abroad $(P<$ 0.05). In terms of occupation, the anxiety score of the staff was higher than that of the overseas students. The anxiety score of entry quarantine personnel from the Asia was higher than that from the Americas, and the anxiety score of the African entry quarantine personnel was higher than that of the American group. In terms of reasons for going abroad, the anxiety score of the quarantined personnel who went abroad for work was higher than those for visiting relatives and studying abroad.

Moreover, there were statistically significant differences in depression scores among subjects with different education levels, occupations, states and reasons for going abroad $(P<0.05)$, In terms of education level, the depression scores of doctoral students, graduate students and undergraduate students were lower than those of specialist and other groups. In terms of occupation, the depression score of staff was higher than that of overseas students. In terms of continent, the depression score of Asian quarantine personnel was higher than that of European and American groups. For the reason of going abroad, the depression score of entry quarantine personnel who went abroad 


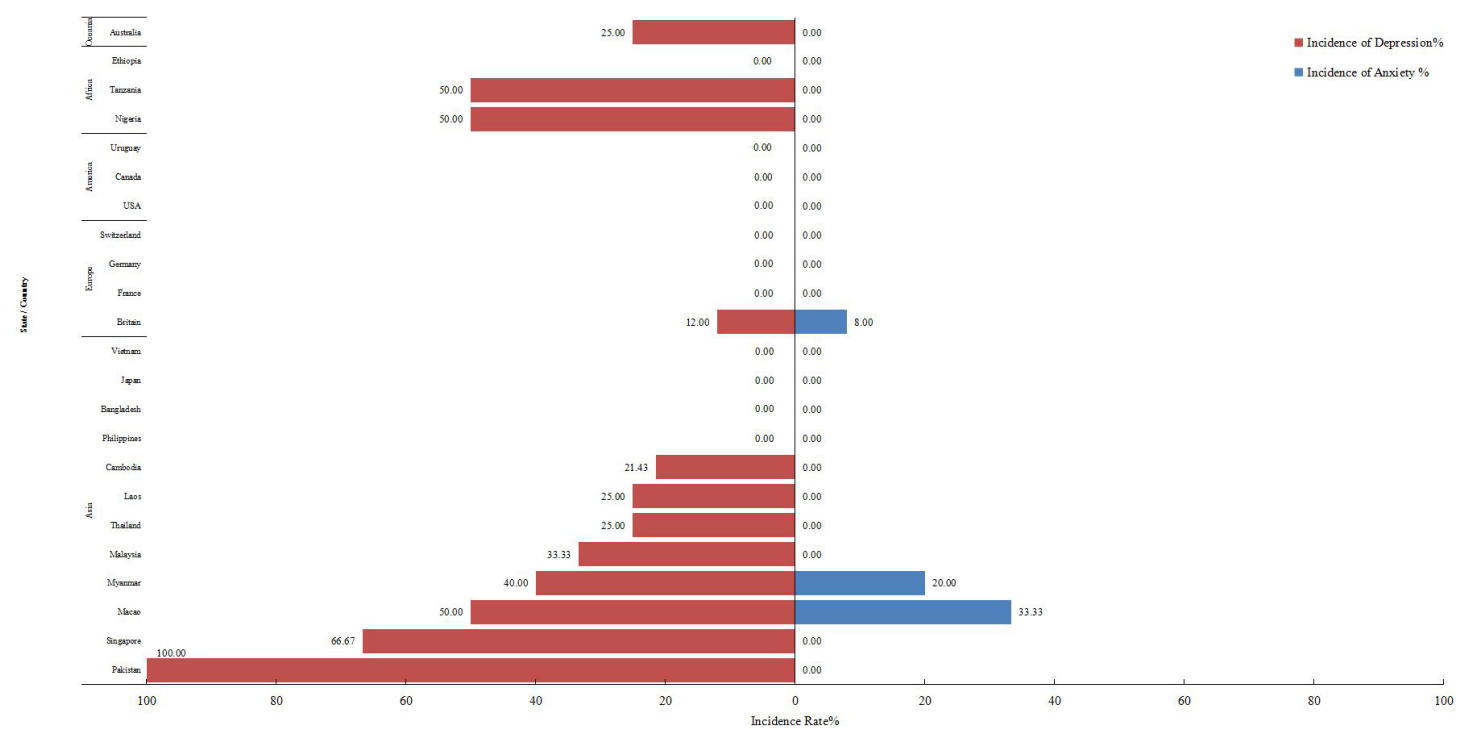

Figure 1. Incidence of anxiety and depression in different continent.

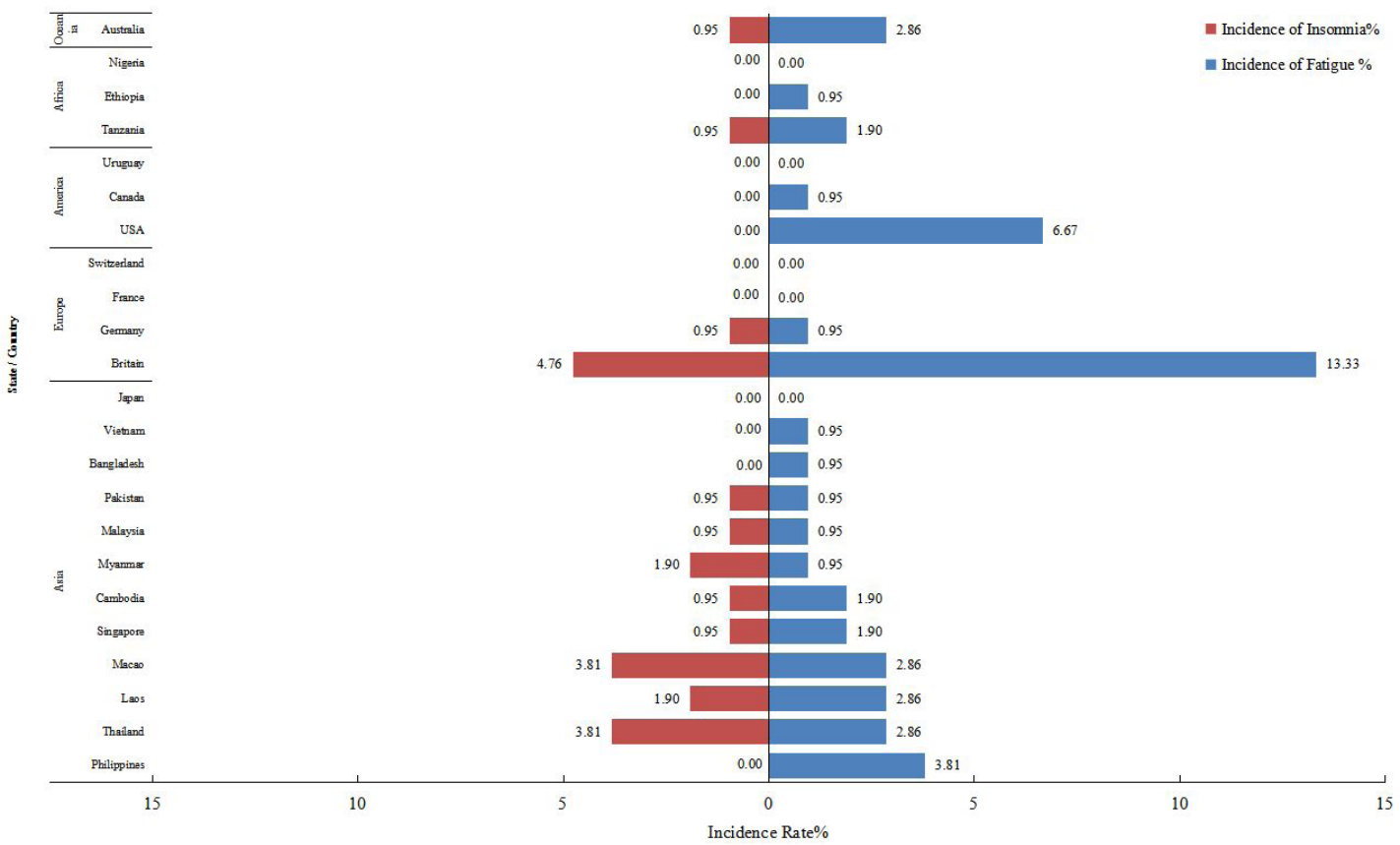

Figure 2. Incidence of fatigue and insomnia in different continents.

for work was higher than that of subjects who went abroad for family visit and study.

However, there were no significant differences in fatigue scores among subjects with different genders, ages, educational levels, occupations, concentrated isolation points, States, reasons for going abroad, and time of living abroad $(P>0.05)$.

Furthermore, there were statistically significant differences in insomnia scores among subjects with different ages and educational levels $(P<0.05)$. In terms of age, the insomnia scores among entry quarantine personnel in the three age groups of 20-29, 30-39 and 40-49 years old were lower than those in the age group of less than 20 years old. In terms of education level, the insomnia scores of entry quarantine personnel who were doctoral students, graduate students and undergraduates were higher than those who were specialty and other groups. The results were shown in Table 4.

\section{Discussion}

The COVID-19 epidemic is spreading globally. At present, China has achieved some results in epidemic prevention and control. However, the imported cases with COVID-19 in Harbin and Mudanjiang, Heilongjiang province, have been warned 
Table 4. Univariate analysis of anxiety, depression, fatigue and insomnia among entry quarantine personnel.

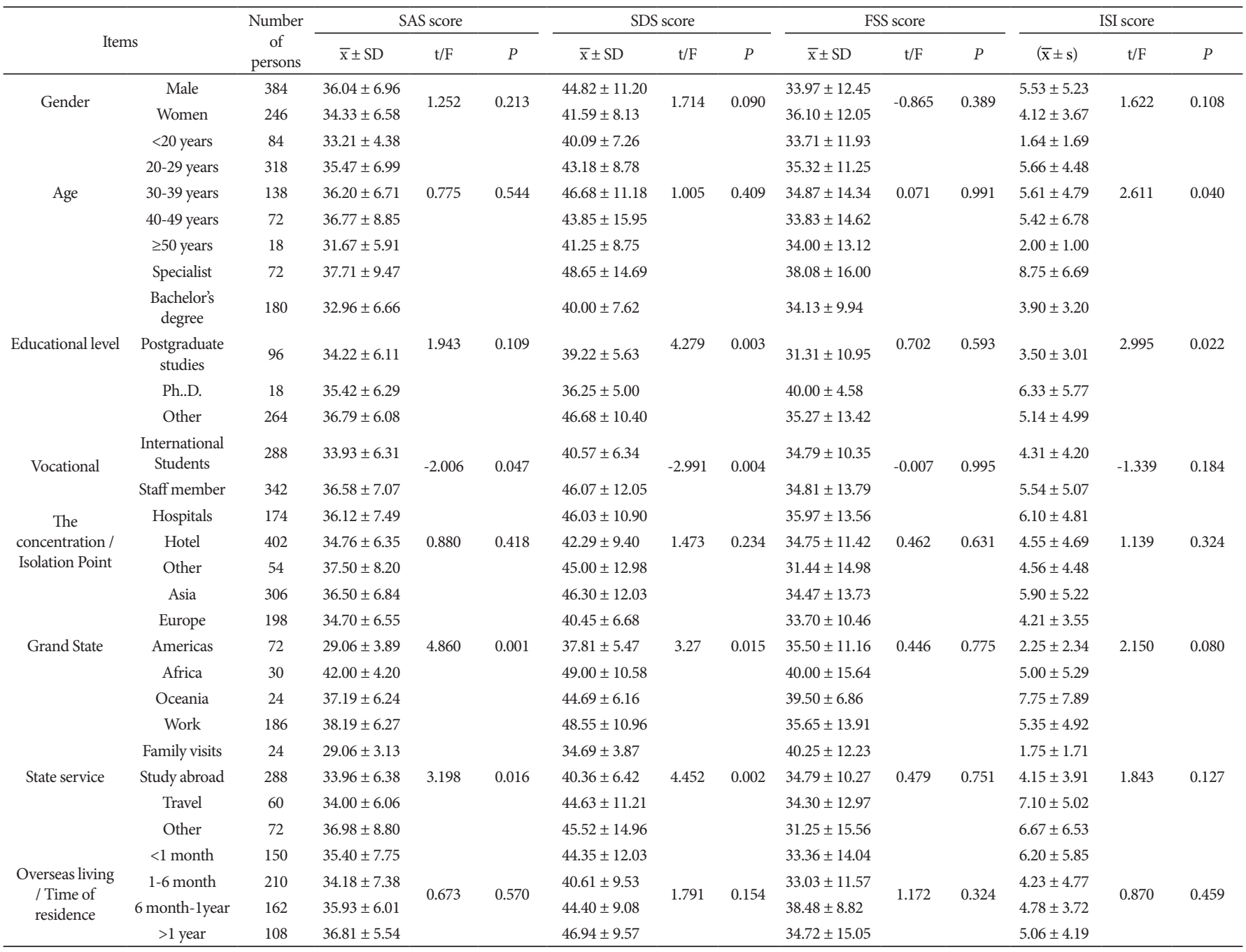

(China, 2020d). Therefore, the epidemic prevention and control still cannot be ignored in China. Since February 29, 2020, China has continuously reported imported cases from abroad. As of June 11,2020, there were 63 confirmed imported cases from abroad, and 1803 cases have been reported. At present, preventing the spread of the COVID-19 epidemic from overseas has become the focus of epidemic prevention and control in China (Qian et al., 2020).

The spread of the COVID-19 has brought different psychological pressure and psychological illness to the community, and has caused different degrees of harm to all kinds of people's lives and psychology (Pan, 2020; Ma \& Yan, 2020; China, 2020c). The entry quarantine personnel are the key population for epidemic prevention and control at present stage. In order to minimize the mental health problems faced by various populations during the development of the epidemic, strengthen psychological counseling and psychological interventions for key populations, help immigrants adapt to the isolation environment, and improve the entry management and service level, the relevant national departments have formulated the $<$ Guiding Principles for Emergency Psychological Crisis Intervention in the Novel
Coronavirus Infected Pneumonia Epidemic $>$ and the $<$ Notice on Strengthening Psychological Assistance and Social Work Services in the Response to the Novel Coronavirus Pneumonia Epidemic> (China, 2020a; Li et al., 2020b).

The age distribution and occupation composition of entry quarantine personnel who entry China are different. Li et al. showed that the age of imported cases tended to be younger, and the majority was overseas students (Sheng et al., 2020). Our results showed that the age of the quarantine personnel was mainly 20-29 years old, and foreign students were the main immigrants. Relevant studies have shown that, in the early stage, Chinese imported cases were mainly imported from ports, and the UK and USA were the main sources (Yang et al., 2020). This survey found that the UK, Cambodia and USA ranked the top three in terms of entry quarantine personnel, and the UK and USA were mainly foreign students, while Cambodia was mainly composed of staff. During the epidemic period, the management of international students from developed countries and Chinese surrounding countries should be strengthened.

The continuous spread of COVID-19 has brought serious psychological pressure to all kinds of people in the society (China, 
2020c). In this survey, 630 entry quarantine personnel all have psychological status, including various degrees of anxiety, depression, fatigue and insomnia. Age, education level, occupation, continent and the reason for going abroad were the main influencing factors of psychological status. The lower the age group, the higher the education level, the healthier the psychological status. The psychological evaluation of international students was healthier than that of staff. The psychological status of Asian entry quarantine personnel was lower than that from some continents. Research by Yang Xinping (Yang et al., 2020) and others showed that the risk of Chinese import from land-based countries bordering China was higher. This survey showed that Asia accounted for a high proportion, which further indicated that the control of inbound personnel in Chinese neighboring countries should be strengthened. Pearson correlation analysis showed that there was a positive correlation between the four indicators among entry quarantine personnel. Relevant studies also showed that sleep quality had a certain correlation with anxiety and depression (Cao et al., 2020), which was similar to the results of ours. The psychosomatic status of entry quarantine personnel is affected by many factors. Previous research showed that sleep status was the influencing factor of anxiety and depression (Chinazzi et al., 2020).

The COVID-19 epidemic situation has been effectively delayed due to Chinese early travel restrictions. More than half of the international output cases have been reduced, which has won valuable time for the international prevention and control measures to cope with the COVID-19 epidemic situation (Anhui, 2020). The Foreign Affairs Office of the people's Government of Anhui Province issued a notice. All personnel from overseas to Anhui (including direct entry from overseas or transit from other cities in China), regardless of Chinese citizens or foreign citizens, shall implement relevant prevention and control measures and strictly implement isolation observation measures. All entry personnel shall implement 14-day centralized isolation medical observation according to relevant regulations Preliminary screening of nucleic acid detection. None of the 630 entry quarantine personnel in this survey was positive detected by nucleic acid test. In order to better prevent and control the follow-up entry quarantine personnel, according to the survey results, we should formulate exercise measures to enhance their immunity, reasonably guide sleep time, and increase the psychological education and training plan for entry quarantine personnel, so as to improve their life experience during the isolation period. Targeted training and psychological counseling measures, such as one-to-one online counseling and other psychological counseling measures, should be carried out to ensure the physical and mental health of the entry quarantine personnel as far as possible.

\section{Conflict of interest}

The author declare that they have no conflict of interest.

\section{Funding}

The second batch of emergency scientific and technological research projects for the prevention and control of pneumonia caused by novel coronavirus pneumonia in Fuyang City: Practical research on infection prevention and control in centralized isolation points for close contacts of novel coronavirus pneumonia (FK 20202804).

\section{References}

Anhui, Foreign Affairs Office of Anhui Provincial People’s Government. (2020). Warm tips for entry (return) personnel to Anhui. Retrieved from http://ahfao.ah.gov.cn/ztzl/yqfk/112270691.html

Cao, J., Wen, M., Shi, Y. R., Wu, Y., \& He, Q. (2020). Prevalence and factors associated with anxiety and depression in patients with Coronavirus disease 2019 (COVID-19). Journal of Nursing Science, 35(09), 15-17.

China, Department of Disease Control and Prevention. (2020a). Notice on strengthening psychological assistance and social work services in response to the outbreak of new coronary pneumonia. Retrieved from http://www.nhc.gov.cn/jkj/s3577/202003/ a9b0bcb3bb7445298c480c5003c51d6d.shtml

China, Medical Administration and Hospital Authority. (2020b). Announcement on the situation of the cluster of new crown pneumonia in Heilongjiang Province. Retrieved from http://www.nhc.gov.cn/ xcs/zhengcwj/202005/9115d2334dfb4b2badcd9a5482f4a9b8.shtml

China, National Health Commission. (2020c). Guiding principles for emergency psychological crisis intervention in the Novel Coronavirus Pneumonia outbreak. Retrieved from http://www.nhc.gov.cn/jkj/s3 577/202001/6adc08b966594253b2b791be5c3b9467.shtml

China, National Health Commission. (2020d). Up to 24 June 11th novel coronavirus pneumonia epidemic situation. Retrieved from http://www.nhc.gov.cn/xcs/yqtb/202106/4c4a185fda3b4e1b85962 34e6a42d731.shtml

Chinazzi, M., Davis, J. T., Ajelli, M., Gioannini, C., Litvinova, M., Merler, S., Piontti, A. P., Mu, K., Rossi, L., Sun, K., Viboud, C., Xiong, X., Yu, H., Halloran, M. E., Longini, I. M. Jr., \& Vespignani, A. (2020). The effect of travel restrictions on the spread of the 2019 novel coronavirus (COVID-19) outbreak. Science, 368(6489), 395-400. http://dx.doi.org/10.1126/science.aba9757. PMid:32144116.

Eurosurveillance Editorial Team. (2020). Note from the editors: World Health Organization declares novel coronavirus (2019-nCoV) sixth public health emergency of international concern. Eurosurveillance, 25(5), 200131e.

Li, E. Z. (2018). The validity and reliability of severe insomnia index scale. Guangdong: Southern Medical University.

Li, Q., Guan, X., Wu, P., Wang, X., Zhou, L., Tong, Y., Ren, R., Leung, K. S. M., Lau, E. H. Y., Wong, J. Y., Xing, X., Xiang, N., Wu, Y., Li, C., Chen, Q., Li, D., Liu, T., Zhao, J., Liu, M., Tu, W., Chen, C., Jin, L., Yang, R., Wang, Q., Zhou, S., Wang, R., Liu, H., Luo, Y., Liu, Y., Shao, G., Li, H., Tao, Z., Yang, Y., Deng, Z., Liu, B., Ma, Z., Zhang, Y., Shi, G., Lam, T. T. Y., Wu, J. T., Gao, G. F., Cowling, B. J., Yang, B., Leung, G. M., \& Feng, Z. (2020a). Early transmission dynamics in Wuhan, China, of Novel Coronavirus-Infected Pneumonia. The New England Journal of Medicine, 382(13), 1199-1207. http://dx.doi. org/10.1056/NEJMoa2001316. PMid:31995857.

Li, Z. H., Wang, J., Huang, J. Y., Lu, J., \& Guo, Z. (2020b). Epidemiological characteristics of imported cases of COVID-19 from outside China in early stage. Journal of Tropical Medicine, 10(29), 04.

Ma, C., \& Yan, X. K. (2020). Research progress in psychological stress expression and prevention and control strategy of COVID-19. Journal of Jilin University (Medicine Edition), 46, 1-6.

Pan, D. L. (2020). Resolutely win the novel coronavirus pneumonia epidemic prevention and control, "psychological warfare". China Health Human Resources, 2020(04), 8-9. Retrieved from: https:// kns.cnki.net $/ \mathrm{kcms} /$ detail/detail.aspx?dbcode $=$ CJFD\&dbname $=$ CJFDLAST2020\&filename $=W S R C 202004004 \& v=i 5 \% 25 \mathrm{mmd} 2$ FjlY5rPJTk1njSgmyFxlFBya2n0Iv0WNQ0TFmdsYDHISAAF4i Bg9dJBWRxzRIE\&UID=WEEvREcwSIJHSldTTEYzWEpEYnlQ 
QmFtODJNUHBzYng0c3MySXhlUnA2ND0\%3d\%249A4hF_YA uvQ5obgVAqNKPCYcEjKensW4IQMovwHtwkF4VYPoHbKxJw !!\&PlatForm $=\mathrm{kdoc}$.

Qian, Y., Hu, S. F., \& Sun, H. Q. (2020). Brief introduction on self-help practical techniques in mentals crisis intervention. Chinese Mental Health Journal, 09(36), 284-285.

Sheng, X., Liu, F., Zhou, J., \& Liao, R. (2020). Psychological status and sleep quality of nursing interns during the outbreak of COVID-19. Nan Fang Yi Ke da Xue Xue Bao, 40(3), 346-350. PMid:32376580.

Wang, X. D., Wang, X. L., \& Ma, H. (1999). Mental health assessment scale manual. Chinese Mental Health Journal, 210-211, 173-174.
Wei, R., \& Song, Y. (2017). Characteristics of fatigue and the effects of sleep behavior intervention on fatigue in patients with Parkinson's disease. Nursing Journal of Chinese People's Liberation Army, 34(23), 39-42.

Yang, X. P., Pang, M. F., Liang, Z. R., Dong, X., Lyu, K., Shi, G., Shi, X., Xi, J., Feng, L., \& Qi, X. (2020). Assessment for the COVID-19 outbreak situation and importation risk of the 14 land-bordering countries of China. Disease Surveillance, 35(04), 283-287.

Yuan, Q., Ma, J. J., Wang, Z. F., Fu, Y., Zhai, J., Feng, Y., Zheng, Y., Guo, Y., \& Liu, Q. (2020). Clinical features and TCM Syndromes Distribution of 41 Patients with New Type Coronavirus Pneumonia imported from abroad in Beijing. World Chinese Medicine, 1-5. 